\title{
Acetophenone Azine: The 2021 American Contact Dermatitis Society Allergen of the Year
}

\author{
Margo Reeder, MD; Amber Reck Atwater, MD
}

\section{PRACTICE POINTS}

- Acetophenone azine is an emerging allergen identified in ethyl-vinyl acetate foam used in shin guards, shoe soles, and flip-flops.

- Cases have been reported in young male athletes in Europe and Canada.

- Patch testing can be completed with acetophenone azine $0.1 \%$ in acetone or petrolatum.

The American Contact Dermatitis Society chose acetophenone azine (AA) as the 2021 Allergen of the Year. Acetophenone azine is an emerging contact allergen that often is associated with the use of sports equipment and footwear. The pattern of dermatitis initially starts locally at the site of contact but can develop into a generalized dermatitis. Strong suspicion is necessary to diagnose AA allergy, and patch testing should be completed with $A A$ and to potentially relevant sports equipment and/or shoes. Acetophenone azine is not yet available as a commercial patch-test preparation, but the optimal concentration is $0.1 \%$ in acetone or petrolatum. This column serves as an introduction to $\mathrm{AA}$ as an emerging allergen and highlights diagnosis, management, and patch testing for AA contact allergy.

t's time for the American Contact Dermatitis Society (ACDS) Allergen of the Year! For 2021, the esteemed award goes to acetophenone azine (AA). If you have never heard of this chemical, you are not alone. Acetophenone azine has been identified in foam materials made of the copolymer ethyl-vinyl acetate (EVA). Contact allergy to AA initially was reported in $2016 .{ }^{1}$ There are only a few European and Canadian case reports and one case series of AA contact allergy in the literature, all of which are associated with foam shin pads or shin guards, shoe insoles, and/or flip-flops. ${ }^{2-6}$ Acetophenone azine is an important emerging allergen, and in this column, we will introduce you to AA and the sneaky places it can lurk and cause allergic contact dermatitis (ACD). We also highlight diagnosis, management, and patch testing for AA contact allergy.

\section{AA Contact Allergy in the Literature}

The first case of AA contact allergy was reported in Europe in 2016 when a 13-year-old male soccer player developed severe lower leg dermatitis and later generalized dermatitis associated with wearing foam shin guards. ${ }^{1}$ Patch testing to standard and supplemental trays was negative or not relevant; however, the patient exhibited strong reactions when patch tested directly to a piece of the shin guard soaked in acetone, water, and ethanol. Additional testing with AA diluted in acetone, water, and petrolatum resulted in positive patch test reactions to acetone dilutions of $1 \%, 0.1 \%, 0.01 \%$, and $0.001 \%$ and aqueous solutions of $1 \%$ and $0.1 \%$. Chromatographic analyses with high-performance liquid chromatography (HPLC) of shin guard extracts confirmed the culprit allergen to be AA. ${ }^{1}$

In the following months, the same clinic saw 2 more cases of AA contact allergy. ${ }^{2}$ An 11-year-old male soccer player developed lower leg dermatitis and later generalized dermatitis from wearing shin guards. Months later,

Dr. Reeder is from the Department of Dermatology, University of Wisconsin School of Medicine and Public Health, Madison. Dr. Atwater is from the Department of Dermatology, Duke University School of Medicine, Durham, North Carolina.

Dr. Reeder is Director of the American Contact Dermatitis Society (ACDS) Contact Allergen Management Program. Dr. Atwater is Immediate Past President of ACDS.

Correspondence: Margo Reeder, MD, 1 South Park St, 7th Floor, Madison, WI 53715 (mreeder@dermatology.wisc.edu). doi:10.12788/cutis.0252 
he also developed dermatitis on the soles of the feet, which was attributed to wearing flip-flops. Patch tests to pieces of the shin guards and flip-flops were positive; $\mathrm{AA}$ in acetone $0.1 \%$ and $0.01 \%$ also was positive. As you might expect, HPLC again confirmed the presence of AA in the shin guards and flip-flops. The third patient was a 12-year-old boy with dermatitis on the soles of both feet; later he also developed a generalized dermatitis. Patch testing to pieces of the insoles of his sneakers and $\mathrm{AA}$ in acetone $0.1 \%$ and $0.01 \%$ was positive. Again, HPLC was positive for the presence of AA in the insoles of his sneakers. ${ }^{2}$

Several more cases of AA contact allergy have been reported in the literature. A 29-year-old European male hockey player demonstrated contact allergy to the gray foam of his shin pads as well as localized leg dermatitis followed by generalized dermatitis (are you noticing a trend yet?), and later dermatitis on the soles of the feet with positive patch-test reactions to pieces of his shin pads and shoe insoles as well as AA $0.1 \%$ and $0.01 \%$ in acetone. ${ }^{3} \mathrm{~A}$ 6-year-old Canadian male soccer player presented with leg dermatitis and later generalized dermatitis and dermatitis on the soles of the feet with positive reactions to pieces of his shin pads and shoe insoles as well as to AA 1\% and $0.1 \%$ in petrolatum. ${ }^{4}$ A 17 -year-old British male (another trend, all males so far!) hockey player developed dermatitis localized to the legs and positive patch tests to the worn foam inner lining of his shin pads as well as to AA $0.1 \%$, $0.01 \%$, and $0.001 \%$ in acetone. ${ }^{5}$

Finally, Darrigade et $\mathrm{al}^{6}$ published a case series of 6 European children with AA contact allergy associated with shin pads and shoes; all had localized leg dermatitis, and some had generalized dermatitis. Patch testing to pieces of shin pads and shoe parts as well as to AA $0.1 \%$ in petrolatum and/or acetone showed with positive reactions to the foam pieces and $\mathrm{AA}$ in all 6 patients.

\section{What's the Deal With AA?}

Acetophenone azine (also known as methylphenylketazine or bis[1-phenylethylidene]hydrazine) is composed of 2 acetophenone structures and a hydrazine moiety. It has been identified in EVA foam, which can be found in sports equipment such as shin pads or shin guards, shoes, and flip-flops. Raison-Peyron et $\mathrm{al}^{1}$ confirmed the presence of AA in EVA foam but reported that they did not know the exact reason for its presence. The authors theorized that AA might be a catalyst during EVA polymerization and also noted that it has antimicrobial and antihelminthic activity. ${ }^{1}$ Several authors noted that AA could be a by-product of EVA synthesis and that sports equipment manufacturers might not be aware of its presence in EVA.,4-6 Some noted that AA concentration was higher in shin guards than in shoe insoles; they thought this explained why patients reacted first to their shin guards and were perhaps even initially sensitized to the shin guards, as well as why shoe insole contact allergy commonly was reported later or only after allergy to shin guards had already developed., ${ }^{4,6}$

\section{Differential Diagnosis of Shin Pad or Shin Guard Dermatitis}

We would be remiss if we did not mention the appropriate differential diagnosis when shin pad or shin guard dermatitis is identified. In fact, in most cases, shin guard dermatitis results from irritant contact dermatitis from friction, heat, and/or perspiration. Acetophenone azine contact allergy is not the most likely diagnosis when your sports-savvy, shin guard-wearing patient presents with anterior lower leg dermatitis. However, when conservative therapy (eg, barrier between the shin guard and the skin, control or management of perspiration, topical corticosteroid therapy) fails, patch testing to evaluate for $\mathrm{ACD}$ is indicated.

\section{Management of AA Contact Allergy}

As astute readers of this column are already aware, treatment of ACD requires strict allergen avoidance. You will find that we have the same recommendations for $\mathrm{AA}$ contact allergy. Given that there are only a handful of cases in the literature, there are limited recommendations on practical allergen avoidance other than "don't wear the problem shin guards, shoe insoles, or flip-flops." However, Darrigade et $\mathrm{al}^{6}$ recommended wearing polyurethane shin guards and leather insoles as alternatives when AA contact allergy is suspected or confirmed. They also made it clear that thick socks worn between shin guards and the skin often are not good enough to avoid ACD because the relevant allergens may achieve skin contact despite the barrier. ${ }^{6}$

\section{Patch Testing for AA Contact Allergy}

Historically, ACD to shin guards or shin pads, insoles of shoes, and even flip-flops has been associated with rubber-related chemicals such as mercapto mix, thiuram mix, $N$-isopropyl- $N^{\prime}$-phenyl-p-phenylenediamine, thioureas, and carbamates, as well as dyes, benzoyl peroxide, and urea formaldehyde or phenol formaldehyde resins. ${ }^{1}$ Most of these chemicals can be tested with standard screening series or supplemental series. Patients with contact allergy to AA may have negative patch testing to screening series and/or supplemental series and may have strong positive reactions to pieces of suspected foam shin pads or shin guards, shoes, and/or flip-flops. Although Koumaki et $\mathrm{al}^{5}$ recommended patch testing for AA contact allergy with AA $0.1 \%$ in acetone, Besner Morin et $\mathrm{al}^{4}$ mentioned that petrolatum may be a more desirable vehicle because it could maintain stability for a longer period of time. In fact, a 2021 article highlighting the American Contact Dermatitis Society Allergen of the Year recommends testing with either AA $0.1 \%$ in acetone or AA $0.1 \%$ in petrolatum. ${ }^{7}$ Unfortunately, AA is not commercially available for purchase at the time of publication. We are hopeful that this will change in the near future.

\section{Final Interpretation}

Acetophenone azine is an emerging allergen commonly identified in EVA foam and attributed to contact allergy to shin guards or pads, soles of shoes, and flip-flops. Most 
cases have been reported in Europe and Canada and have been identified in young male athletes. In addition to standard patch testing, athletes with lower leg dermatitis and/ or dermatitis of the soles of the feet should undergo patch testing with AA $0.1 \%$ in acetone or petrolatum and pieces of the equipment and/or footwear.

\section{REFERENCES}

1. Raison-Peyron N, Bergendorff O, Bourrain J, et al. Acetophenone azine: a new allergen responsible for severe contact dermatitis from shin pads. Contact Dermatitis. 2016;75:106-110.

2. Raison-Peyron N, Bergendorff $\mathrm{O}$, Du-Thanh A, et al. Two new cases of severe allergic contact dermatitis caused by acetophenone azine. Contact Dermatitis. 2017;76:380-381.
3. De Fré C, Bergendorff $\mathrm{O}$, Raison-Peyron N, et al. Acetophenone azine: a new shoe allergen causing severe foot dermatitis. Contact Dermatitis. 2017;77:416-417.

4. Besner Morin C, Stanciu M, Miedzybrodzki B, et al. Allergic contact dermatitis from acetophenone azine in a Canadian child. Contact Dermatitis. 2020;83:41-42.

5. Koumaki D, Bergendorff $\mathrm{O}$, Bruze $\mathrm{M}$, et al. Allergic contact dermatitis to shin pads in a hockey player: acetophenone is an emerging allergen. Dermatitis. 2019;30:162-163.

6. Darrigade AS, Raison-Peyron N, Courouge-Dorcier D, et al. The chemical acetophenone azine: an important cause of shin and foot dermatitis in children. J Eur Acad Dermatol Venereol. 2020;34:E61-E62.

7. Raison-Peyron N, Sasseville D. Acetophenone azine. Dermatitis. 2021;32:5-9. 